\title{
Non-intubated thoracic surgery under thoracic epidural anesthesia
}

\author{
Sung Yong Park \\ Department of Anesthesiology and Pain Medicine, Ajou University School of Medicine, Suwon, Korea
}

Traditionally, one-lung ventilation (OLV) using a doublelumen endotracheal tube or bronchial blocker with general anesthesia is the first anesthetic choice in thoracic surgery. However, general anesthesia with mechanical ventilation carries a risk of side-effects related to tracheal intubation and general anesthesia, such as airway complications, ventilation-induced lung injury, and residual neuromuscular blockade [1-3]. Thanks to advances in minimally invasive techniques, non-intubated thoracic surgery with spontaneous ventilation provides another anesthetic option for high-risk patients who may not tolerate intubated general anesthesia, and avoids complications related to intubation and OLV [4]. Although still relatively uncommon, a few randomized trials [5-8] and a meta-analysis [9] have indicated that non-intubated thoracic surgery with spontaneous ventilation is more physiological and has more advantages than mechanical ventilation.

The current issue of the Korean Journal of Anesthesiology [10] presents a case report on non-intubated thoracoscopic surgery under thoracic epidural anesthesia. The authors showed that thoracic epidural anesthesia with a target-controlled infusion of remifentanil and propofol for sedation was a feasible strategy for thoracoscopic surgery in patients with complicated respiratory function.

To ensure patient safety, anesthetic considerations for respiratory management are essential. After the parietal pleura is opened during spontaneous breathing, the lung will collapse on exposure to atmospheric pressure, and OLV will begin [11]. Regional anesthetic techniques, such as thoracic epidural anesthesia (level T3-T4, sensory blockade T2-T10), paravertebral block, or intercostal block are effective for performing numerous procedures in thoracic surgery, with the patients awake or under minimal sedation [12]. However, stimulation of the visceral pleura, bronchi, or trachea may trigger the cough reflex. Such visceral afferent transmissions cannot be blocked by epidural anesthesia or intercostal block. Many approaches have been proposed to control the cough reflex, including intrathoracic vagal and phrenic nerve block, stellate ganglion block, and intravenous administration of opioids [13].

Sometimes, intraoperative conversion to general anesthesia is inevitable because of major bleeding, dense adhesions, insufficient analgesia, inadequate sedation, or persistent hypoxemia $[12,13]$. The anesthesiologist must have a protocol to minimize the risk to the patient before the operation. Intubation in the lateral decubitus position is a technical challenge and may result in critical complications [12]. A single-lumen tube is usually recommended for endotracheal intubation at the time of case conversion. After airway control has been achieved, a bronchial blocker can be used to obtain OLV [11].

Although the benefits remain unclear, non-intubated thoracic surgery may be preferable over general anesthesia with endotracheal intubation for specific indications. The anesthetic team must be aware of the potential problems, and have familiarity with the procedure to be performed. Education and training programs in thoracic surgery with non-intubated patients may be needed.

Corresponding author: Sung Yong Park, M.D.

Department of Anesthesiology and Pain Medicine, Ajou University School of Medicine, 206, World cup-ro, Yeongtong-gu, Suwon 16499, Korea Tel: 82-31-219-5573, Fax: 82-31-219-5579

Email: anepark@hanmail.net

ORCID: http://orcid.org/0000-0003-2395-1645

Korean J Anesthesiol 2017 June 70(3): 235-236

https://doi.org/10.4097/kjae.2017.70.3.235

(c) This is an open-access article distributed under the terms of the Creative Commons Attribution Non-Commercial License (http://creativecommons.org/ licenses/by-nc/4.0/), which permits unrestricted non-commercial use, distribution, and reproduction in any medium, provided the original work is properly cited. 


\section{References}

1. Serpa Neto A, Hemmes SN, Barbas CS, Beiderlinden M, Biehl M, Binnekade JM, et al. Protective versus conventional ventilation for surgery: a systematic review and individual patient data meta-analysis. Anesthesiology 2015; 123: 66-78.

2. Neto AS, Hemmes SN, Barbas CS, Beiderlinden M, Fernandez-Bustamante A, Futier E, et al. Association between driving pressure and development of postoperative pulmonary complications in patients undergoing mechanical ventilation for general anaesthesia: a metaanalysis of individual patient data. Lancet Respir Med 2016; 4: 272-80.

3. Murphy GS, Szokol JW, Marymont JH, Greenberg SB, Avram MJ, Vender JS. Residual neuromuscular blockade and critical respiratory events in the postanesthesia care unit. Anesth Analg 2008; 107: 130-7.

4. Pompeo E, Mineo D, Rogliani P, Sabato AF, Mineo TC. Feasibility and results of awake thoracoscopic resection of solitary pulmonary nodules. Ann Thorac Surg 2004; 78: 1761-8.

5. Pompeo E, Tacconi F, Mineo D, Mineo TC. The role of awake video-assisted thoracoscopic surgery in spontaneous pneumothorax. J Thorac Cardiovasc Surg 2007; 133: 786-90.

6. Pompeo E, Rogliani P, Tacconi F, Dauri M, Saltini C, Novelli G, et al. Randomized comparison of awake nonresectional versus nonawake resectional lung volume reduction surgery. J Thorac Cardiovasc Surg 2012; 143: 47-54.e1.

7. Pompeo E, Dauri M. Is there any benefit in using awake anesthesia with thoracic epidural in thoracoscopic talc pleurodesis? J Thorac Cardiovasc Surg 2013; 146: 495-7.e1.

8. Liu J, Cui F, Li S, Chen H, Shao W, Liang L, et al. Nonintubated video-assisted thoracoscopic surgery under epidural anesthesia compared with conventional anesthetic option: a randomized control study. Surg Innov 2015; 22: 123-30.

9. Tacconi F, Pompeo E. Non-intubated video-assisted thoracic surgery: where does evidence stand? J Thorac Dis 2016; 8(Suppl 4): S364-75.

10. Moon E, Go Y, Chung J, Yi J. Non-intubated thoracoscopic surgery for decortication of empyema under thoracic epidural anesthesia. Korean J Anesthesiol 2017; 70: 341-4.

11. Sunaga H, Blasberg JD, Heerdt PM. Anesthesia for nonintubated video-assisted thoracic surgery. Curr Opin Anaesthesiol 2017; $30: 1-6$.

12. Gonzalez-Rivas D, Bonome C, Fieira E, Aymerich H, Fernandez R, Delgado M, et al. Non-intubated video-assisted thoracoscopic lung resections: the future of thoracic surgery? Eur J Cardiothorac Surg 2016; 49: 721-31.

13. Iwata Y, Hamai Y, Koyama T. Anesthetic management of nonintubated video-assisted thoracoscopic surgery using epidural anesthesia and dexmedetomidine in three patients with severe respiratory dysfunction. J Anesth 2016; 30: 324-7. 